\title{
Adoption of Immersive Technologies in Manufacturing SMEs - A Strategy- as-Practice Perspective on their Affordances, Constraints and Responses
}

\author{
Charlotte Wendt \\ Technical University of Darmstadt \\ wendt@ise.tu-darmstadt.de
}

\author{
Bradley Kalgovas \\ Technical University of Darmstadt \\ kalgovas@ise.tu-darmstadt.de
}

\author{
Alexander Benlian \\ Technical University of Darmstadt \\ benlian@ise.tu-darmstadt.de
}

\begin{abstract}
Immersive technologies (ImT) provide affordances for small and medium-sized enterprises (SMEs) in the manufacturing industry, such as the enhancement and recreation of aspects of the physical world. These affordances are critical due to changes in the SMEs' environment including increasingly virtualized collaboration and digitization of work practices. However, the adoption of ImT by manufacturing SMEs is still low. Drawing on the Technology Affordances and Constraints theory, and applying a Strategy-as-Practice approach, this study researches the adoption of $\operatorname{Im} T$ as a strategic response to environmental changes in German-based manufacturing SMEs. The findings reveal the critical role of constraints of $\operatorname{Im} T$, which act as hindrances to implementation. Specifically, realizing the affordances of ImT requires consideration of, and responses to, the constraints manufacturing SMEs experience, including the constraints associated with the providers of ImT. Thus, this paper expands the understanding of the factors which influence implementation and strategizing of ImT in SMEs.
\end{abstract}

\section{Introduction}

Immersive technologies (ImT), including virtual reality (VR) and augmented reality (AR) technologies, are increasingly important to intentionally change or enhance an individual's perception of the reality $[1,2]$ and to create an immersive and personalized experience $[3,4]$. While consumer use cases comprise the majority of global spending on ImT, business use cases (e.g., in manufacturing) show strong compound growth rates in excess of $90 \%$, with US $\$ 8$ billion forecasted to be invested in training and industrial maintenance use cases in 2024 [3]. The current demand growth is based on ImT's value to enterprises, consisting of affordances which include enhancement or recreation of the physical world's positive aspects, reduction of the negative aspects, or the creation of aspects that do not exist in the physical world $[2,5,6] . \operatorname{ImT}$ "change the ways we learn, accomplish tasks, and interact with the world" $[2, \mathrm{p}$. 684] and deliver improved business performance through enhanced collaboration outcomes and cost efficiencies. More specifically, employees in manufacturing settings can receive information in their field of view by ImT, allowing for hands-free usage and therefore supporting tasks that benefit from virtual guidance [7]. However, the enterprise adoption rate of $\operatorname{ImT}$ is still low. Prior literature indicates factors which hinder adoption and are on both the adopter and provider side: Adopting ImT requires that relevant infrastructure and hardware is provided by the adopting enterprise, and that employees are familiar with ImT and their features $[2,8,9]$. With respect to the providers, many $\operatorname{ImT}$ are still considered immature as they often have technological weaknesses (e.g., insufficient precision of gesture tracking) or are inconvenient for users (e.g., poor ergonomics and wearability) [7, 9].

Factors that inhibit adoption, hereafter referred to as constraints, are of particular relevance for small- and medium-sized enterprises (SMEs) or enterprises that fall into the comparable category of family-owned firms [10]. These enterprises account for the majority of enterprises worldwide across all industries, including manufacturing $[10,11]$. For the sake of clarity, both SMEs and family-owned firms will be denoted by the term SMEs, as there is substantial overlap [12]. Even though family firms have more employees and annual turnover than SMEs typically have [12], they share many characteristics on organizational and managerial levels $[10,13]$. More specifically, both SMEs and family-owned firms typically face limitations regarding financial resources and access to a broad range of capabilities, have a flat hierarchy, have greater flexibility in decision making, and have a strong ownermanager role $[10,13,14]$. These organizational characteristics significantly impact an SME's adoption of technology [10, 14, 15], particularly when technologies are implemented as a strategic response to environmental changes such as a shift towards virtual work forces [16]. This requires the strategy's acceptance by those who 'do' the strategy internally (i.e., 
management and employees), as well as those who are external stakeholders (i.e., customers and suppliers) [17, $18,19]$. SMEs are less likely to be aware of existing ImT and their respective affordances in the manufacturing context due to limited financial resources and often smaller information technology and research departments $[14,15,20]$. Well-known ImT affordances that have been analyzed for enterprises of different sizes and from different industries [2], do not necessarily apply to manufacturing SMEs. Furthermore, while most research on ImT has focused on the affordances $[2,5$, $6]$, this is only one viewpoint and omits constraints which potentially hinder technology adoption [21]. Prior research indicates that such constraints cause individuals to change aspects of their environment or the applied technology [22]. Consequently, not only affordances but also constraints, and responses that address the constraints, should be considered when studying adoption of ImT. Therefore, this study investigates the following research questions:

RQ1: What affordances are provided by $\operatorname{ImT}$ to manufacturing SMEs?

RQ2: What constraints associated with ImT impact the adoption of ImT by manufacturing SMEs?

RQ3: How can manufacturing SMEs and providers of ImT respond to existing constraints of ImT?

Our study extends previous research focused on the affordances of ImT [2] and applies a Strategy-asPractice (SaP) approach (i.e., an activity-based approach bringing human actions into the centre of strategy research [23]) to investigate affordances that are specific to manufacturing SMEs. To achieve this, interviews were conducted with providers of ImT and with SMEs that already use or are considering the adoption of ImT in manufacturing contexts. By combining the provider and adopter perspectives, the study contrasts the affordances of ImT with their constraints and analyzes the responses of providers and adopters who address the respective constraints as a strategic response.

The findings contribute to research on affordances and constraints of ImT, as well as strategizing in manufacturing SMEs in two important ways. Firstly, by uncovering and contrasting ImT's affordances and constraints for manufacturing SMEs, this research contributes to a more holistic understanding of the enabling and inhibiting factors for the adoption of ImT. Secondly, by considering SMEs' specific characteristics and dependence on ImT providers, the study enhances the understanding of the adoption of ImT as a strategic response and contributes to a more encompassing view of $\mathrm{SaP}$ in SMEs. Moreover, the study identifies practical implications for executives of manufacturing SMEs who consider adoption of ImT as a strategic response to environmental changes by providing an overview on affordances, potential constraints, as well as the respective responses, that can be taken by the SMEs themselves and providers, to successfully adopt ImT.

\section{Theoretical foundations}

\subsection{Immersive technologies}

ImT such as VR, AR, and virtual assistants, have been discussed since the 1960s [9, 24] but have recently received more attention due to the release of more affordable devices (e.g., head-mounted displays from suppliers like HTC and Oculus) [4]. Generally, ImT incorporate "a set of technologies that enable people to immersively experience a world beyond reality" [9, $\mathrm{p}$. 2]. Critical to $\operatorname{ImT}$ is the interaction of individuals with their virtual environment which is enabled by technologies such as movement and gesture recognition algorithms, real-time computing, as well as hardware including controllers, goggles, and haptic devices [9]. Whereas AR technologies enhance certain aspects of an individual's real world environment by means of virtual objects, VR technologies can enable individuals to be fully immersed in a virtual world and completely forget about their real world environment [1, 4]. However, boundaries between both are blurring, and VR and AR are complemented by Mixed Reality $[1,8]$. Consequently, ImT cover a whole virtuality continuum that connects completely real to virtual environments [1]. Accordingly, different ImT present different degrees of virtuality, depending on aspects such as the user's awareness of the surrounding world or whether the user is interacting with virtual or real objects $[1,8]$.

Many different manufacturing applications for ImT have been identified, including product design and development, communication and collaboration across disciplines or in virtual settings, decision-making, as well as training and learning environments [9, 25]. Specifically, ImT are particularly relevant in situations or for tasks with high information density (e.g., maintenance tasks of service technicians) [7], for activities that are impossible or dangerous to practice in physical reality (e.g., emergency scenarios) [2, 26], or when hands-free instructions are required (e.g., assembly tasks performed by one technician alone) [7].

\subsection{Strategy-as-Practice as an overarching lens}

$\mathrm{SaP}$ is an activity-based approach concerned with the day-to-day activities of managers and other actors within and related to the organization, thereby "bringing human actors and their actions and interactions to the centre stage of strategy research" [23, p. 70]. According to $\mathrm{SaP}$ research, strategy is "a situated, socially accomplished activity, while strategizing comprises 
those actions, interactions, and negotiations of multiple actors and the situated practices that they draw upon in accomplishing that activity" [17, p. 7-8]. In doing so, strategy and strategizing are often viewed through the activities undertaken at different levels (i.e., institutional, organizational, and actors) from a processes and content perspective [17].

$\mathrm{SaP}$ is a useful approach to examine situations in which the strategy is emergent and evolving in response to changing environmental, organizational and technological needs $[17,18]$. The strategic response to environmental changes (e.g., digitization of work practices and COVID-19-related travel restrictions) can result in the decision to adopt ImT. According to SaP, strategic decisions require organizational level commitment (i.e., organizational strategy) but also comprise implications on the institutional level (i.e., customer and supplier strategy), and on the level of individual actors within that organization (i.e., actors' routines and activities) who make sense and give sense to other organizational actors [17]. These dynamics are heightened in an SME environment where strategy and practice are often intertwined and indistinguishable.

\subsection{Technology affordances and constraints theory}

Even though the concept of affordances originates in ecological psychology [27], it has been transferred successfully to IS research [28] and has since then been applied to different technologies such as enterprise systems [21], video-conferencing tools [29], and also $\operatorname{ImT}[2,8]$. Affordances are "the possibilities for goaloriented action afforded to specified user groups by technical objects" [30, p. 622]. Technologies are often perceived by actors (i.e., individuals, groups, or enterprises) as sets of affordances and are perceived differently depending upon the actor's goals and abilities as well as the technology's features [2, 22]. Generally, affordances of ImT include enhancing or recreating positive aspects of the physical world, diminishing negative aspects, or creating aspects that do not exist in the physical world, thereby supporting collaboration and communication, and allowing for physical interaction with digital objects [2]. More specifically in manufacturing environments, ImT can afford engineers spatial cognition, accelerated feedback loops [5], or immersion, presence, and empathy in learning environments [6].

Constraints are the counterpart to affordances and typically arise from the absence of a desired affordance, from affordances that are inappropriately realized and thus not perceived as an affordance, or from individuals who are unwilling or unable to realize an affordance [21]. Therefore, constraints potentially deter technology adoption, cause implementation difficulties or, in other words, cause the actor to perceive "that [the technology] constrains their ability to carry out their goals" [22, p. 153]. In IS research, constraints have been examined less frequently than affordances, but are, at times, addressed indirectly when discussing 'disadvantages', 'tensions' or 'weaknesses'. For example, Kammler et al. [7] elaborated on the disadvantages and weaknesses of $\mathrm{AR}$ in mechanical engineering, such as blurry visuals and limited field of view, which can also be analyzed as the constraints of AR for technicians.

\section{Research methodology}

\subsection{Research design}

To answer the research questions, this study employed a qualitative exploratory case study design, which is particularly suitable to develop an accurate picture of the situation and its implications and to elaborate a new framework or theory [31, 32]. For this purpose, a key informant approach was utilized and expert interviews were conducted with executives and project managers of $7 \mathrm{SMEs}$ that offer or promote ImT, as well as 10 manufacturing SMEs that are interested in, or have already adopted, ImT in their manufacturing environments [33]. The inclusion of multiple perspectives enabled broader exploration of the research questions and the underlying "how" and "why" of the observed phenomena as well as more generalizable findings [31, 34]. Consequently, a qualitative approach was applied to extend existing research on affordances related to $\operatorname{ImT}$ and to investigate simultaneously occurring constraints to better understand strategic responses that enable the adoption of $\operatorname{ImT}$ in manufacturing SMEs.

\subsection{Sample description and data collection}

The interviewees were determined by purposive sampling, a non-random sampling technique that utilizes the intentional selection of participants based on the researchers' assessment of the potential participants' experience, knowledge, and willingness to contribute $[10,35]$. Specifically, the interview partners were identified by conducting a web-search for SMEs that either offer or apply immersive systems in a manufacturing context (see Table 1 for details). The study is focused on manufacturing SMEs with German headquarters as many are known as 'hidden champions' that operate in a global market and rely on interaction with internal and external stakeholders in different geographies. Such interaction is increasingly 
virtualized, for instance to minimize travel efforts or to improve employee acquisition and satisfactions $[2,16]$.

All of the interviews took place between November 2020 and January 2021 and were conducted via videocall. The interviews were conducted in a semi-structured manner which allowed the natural views of the interviewees to be captured [36, 37]. The interview guide's scripted questions incorporated openness and flexibility [36], and comprised the dimensions of the organization (i.e., SMEs' characteristics, specificities and goals), the $\operatorname{ImT}$ (i.e., their features, affordances, and constraints) and the SMEs' environment (i.e., industry and market trends). The guiding questions for each dimension were slightly tailored to the respective group of interview partners (i.e., providers vs. adopters).

Table 1. Overview of interview partners

\begin{tabular}{|l|l|l|l|l|}
\hline ID & Focus & $\begin{array}{l}\text { Emplo- } \\
\text { yees }\end{array}$ & $\begin{array}{l}\text { Revenues } \\
{[\mathbf{m} \text { EUR }]}\end{array}$ & $\begin{array}{l}\text { Interviewee } \\
\text { position }\end{array}$ \\
\hline I01 & Provider & $1-9$ & $1-5$ & MD \\
\hline I02 & Provider & $10-49$ & n/a & PM \\
\hline I03 & Provider & $1-9$ & n/a & MD \\
\hline I04 & Provider & $10-49$ & $1-5$ & MD \\
\hline I05 & $\begin{array}{l}\text { VR/AR } \\
\text { research }\end{array}$ & $10-49$ & n/a & Researcher \\
\hline I06 & Adopter & $10-49$ & n/a & DM \\
\hline I07 & Adopter & $50-99$ & $10-50$ & DM \\
\hline I08 & Provider & $20 \mathrm{k}-24 \mathrm{k}$ & $>1,000$ & $\begin{array}{l}\text { Sales } \\
\text { employee }\end{array}$ \\
\hline I09 & $\begin{array}{l}\text { Manufac. } \\
\text { association }\end{array}$ & $500-999$ & n/a & DM \\
\hline I10 & Adopter & $10 \mathrm{k}-14 \mathrm{k}$ & $>1,000$ & DM \\
\hline I11 & Adopter & $5 \mathrm{k}-9 \mathrm{k}$ & $>1,000$ & $\begin{array}{l}\text { Chief Sales } \\
\text { Officer }\end{array}$ \\
\hline I12 & Adopter & $5 \mathrm{k}-9 \mathrm{k}$ & $>1,000$ & $\begin{array}{l}\text { Innovation } \\
\text { manager }\end{array}$ \\
\hline I13 & Adopter & $1 \mathrm{k}-4 \mathrm{k}$ & $>1,000$ & PlM \\
\hline I14 & Adopter & $500-999$ & $100-500$ & 2 MDs \\
\hline I15 & Adopter & $10 \mathrm{k}-14 \mathrm{k}$ & $>1,000$ & MD \\
\hline I16 & Adopter & $100-249$ & $10-50$ & PlM \\
\hline I17 & $\begin{array}{l}\text { Adopter/ } \\
\text { consultant }\end{array}$ & $\mathrm{n} / \mathrm{a}$ & $\mathrm{n} / \mathrm{a}$ & PM \\
\hline MD $=$ Managing director, PM = Project manager, \\
DM = Department manager, PlM = Plant manager
\end{tabular}

\subsection{Data analysis}

The audio-recorded interviews were transcribed, following the established approaches for qualitative data analysis. Two researchers independently conducted thematic coding, using the methodology of data reduction, data display and conclusion drawing [38]. During data reduction, interviews were analyzed with systematic coding procedures, using the NVivo software to assist with the coding and analysis process. Building on technology affordances and constraints theory which states that a technology's affordances and constraints rely on the actors' goals and abilities, the list of initial codes contained the pre-defined categories "goals", "affordances" and "constraints" per actor. Moreover, codes for ImT affordances were determined in advance based on previously researched affordances of $\operatorname{ImT}$ (deductive), which were extended by subaffordances, constraints, and responses in a data-driven coding procedure (inductive) [39]. Subsequently, the codes were clustered into categories and sub-categories according to recurrent patterns and linked to each other, enabling analysis of themes and their interrelations, leading toward refinement of the findings [38, 39]. This was an iterative process with further episodes of data reduction occurring to validate the themes and elaborate on the clusters [38]. During the data display stage, the data was visualized through the hierarchy of codes and categories that were created in NVivo to refine the emerging SaP-based framework. Throughout the final stage, conclusions were drawn by noting patterns and elaborating on causal flows and propositions [38]. Data analysis started after the first interview and continued after each interview until no new insights were generated (i.e., saturation was reached) [40].

\section{Results}

Providers of ImT and manufacturing SMEs (i.e., ImT adopters) are focused on increasing the adoption rate of $\operatorname{ImT}$. Figure 1 depicts the strategic decision stages related to ImT adoption, which in turn impacts the actual implementation of ImT. Specifically, there are two strategy loops, one for ImT providers and one for manufacturing SMEs whose goals initiate the loops while the ImT's affordances act as a motivator to complete the process. Within each loop there is an analysis of the constraints and the associated responses. The results reveal how the goals of manufacturing SMEs can be achieved by realizing the affordances of $\operatorname{ImT}$ and what role constraints and respective responses from providers and manufacturing SMEs play in this context. 


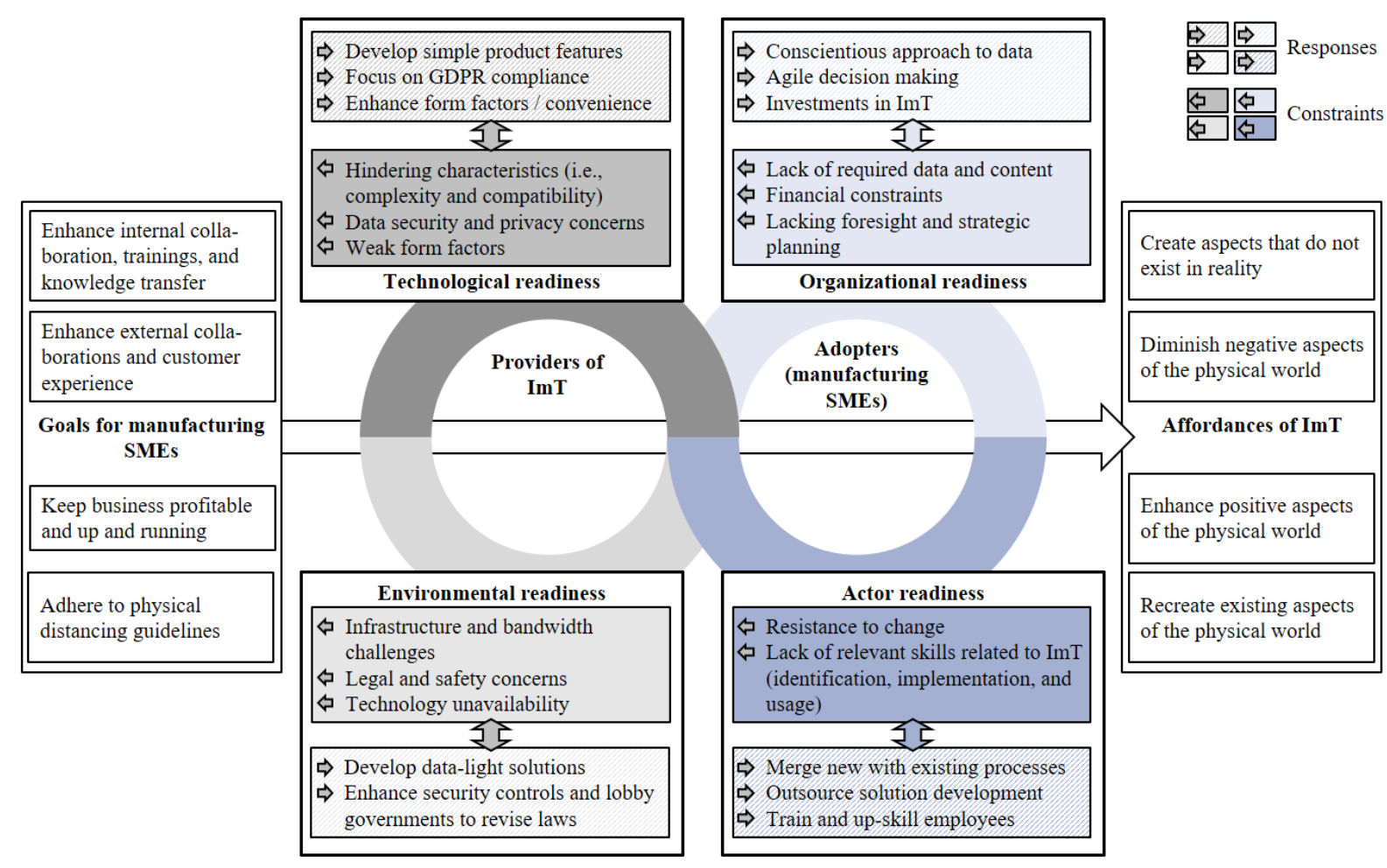

Figure 1. Affordances and constraints of $\operatorname{ImT}$ and responses by providers and adopters

\subsection{Goals for manufacturing SMEs}

Manufacturing SMEs considering the adoption of $\operatorname{ImT}$ do so in pursuit of four main goals. Firstly, they aim to enhance internal collaboration, trainings, and knowledge transfer. Interviews reveal that manufacturing SMEs are looking for ways to improve how employees interact with their globally dispersed colleagues, including in machinery development, set up, and maintenance. Previously, employees were required to undertake significant travel or deal with language barriers and work across different time zones. However currently, SMEs are looking for technology-based ways to enable efficient employee collaboration and knowledge transfer.

Manufacturing SMEs also aspire to enhance external collaboration (e.g., with customers and suppliers) and customer experience. They want to overcome the physical limitations and costs often associated with external collaboration. Specifically, machinery planning and installation is often a key event where onsite presence is required to set up the machine and to transfer knowledge about its use. Also, there are often questions or troubleshooting required to maintain the machinery once it is commissioned.

Moreover, manufacturing SMEs consider ImT adoption to keep their business profitable and up and running. Like other SMEs, manufacturing SMEs are cost conscious and aim to reduce costs associated with their operations, thus increasing business profitability. These costs are often significant when expertise is required which is not located on site, or when large amounts of information need to be easily and rapidly accessible to keep a production line online.

Finally, the environmental conditions have changed over the course of the COVID-19 pandemic, resulting in SMEs aiming to adhere to physical distancing guidelines and to facilitate remote work. COVID-19 has had significant effects on the ability of manufacturing SMEs to effectively and efficiently continue production. Specifically, numerous governments have required enterprises to ensure physical distancing and released strict travel restrictions that have caused the need to adjust, for instance when installing and maintaining machinery: "What got a huge boost with this remote support [...] People can't travel because the planes don't fly, but they still have to maintain the machines, repair them, put them into operation, assemble" [I01].

\subsection{Affordances of immersive technologies}

Numerous affordances are provided by $\operatorname{ImT}$ to achieve the previously mentioned goals. Specifically, there are four key themes for manufacturing SMEs: 
ImT afford manufacturing SMEs' employees to create aspects that do not exist in reality by simulating environments and thus, enabling collaboration and interaction in immersive reality. The capacity to forecast responses, or even incidents, also affords the opportunity to conduct risk-free testing and avoid costly mistakes. Typically, ImT also afford overcoming the space time linearity, for instance by identifying bottlenecks in the manufacturing floor. This is also an approach to assist in sales activities, affording customers to remotely interact with their future products early on (e.g., during the product development). The customer can walk through a virtual show room, virtually loading the machine, and simulating the production process. The ImT also afford product adjustments based on customer feedback.

Moreover, ImT afford the potential to diminish negative aspects of the physical world by reducing the physical risk associated with working in a realworld environment. Especially in the manufacturing environment, simulating situations with $\operatorname{ImT}$ affords risk reduction to those undertaking work in potentially hazardous settings. As an example, "engineers can be trained on a machine they have never used before by [...] seeing in AR how the machine fundamentally works. Then they already have an initial education and know how to handle the machine" [I04].

ImT afford the enhancement of positive aspects of the physical world, especially with AR when objects can be superimposed over the physical reality. This is often vital in manufacturing contexts to afford efficient collaboration and communication. Related use cases have gained significant attention by ImT providers due to the increased need for remote collaboration between different enterprises and organizational functions, especially when they are geographically separated. In the case of commissioning and remote maintenance, the service technician may be located in Europe, while the client, located in a different country, requires real-time assistance. ImT can also afford the bridging of language barriers using visual communication. Moreover, ImT assist manufacturing SMEs' employees in filtering information and afford the provision of context-specific information that is relevant to the current situation. For example, an ARenabled phone held over a broken machine can provide instructions to the mechanic on adjustments required which affords simplification and counters the information overload that is created in the physical world: "People came to us and said that they have been working here for 5 years, but have never figured out why they are setting certain parameters on the machine. With the help of [ImT], we were able to teach them why it needs to be done" [I05].

Moreover, ImT can afford to recreate existing aspects of the physical world, including the emotional interactions among people. More specifically, ImT can help in cases of geographical separation where close interaction is essential (e.g., interactive team settings, team building tasks). Particularly in the manufacturing context, there is also the physical interaction with digital objects where the ability of $\operatorname{ImT}$ to recreate objects affords interaction with their digital twins: "We create a digital twin and you can basically see your production on this system before the machine is built" [I11]. In this way, ImT also afford reduced resource costs (e.g., costs of the employees' time or travel expenses) as they enable remote commissioning and maintenance. ImT also afford advanced learning and training environments. For instance, service team members can be virtually trained on machines, with the advantage of a multisensory, safe, and realistic training experience.

\subsection{Constraints of immersive technologies and corresponding responses}

In order to unleash the affordances of $\operatorname{ImT}$ and thus, to achieve their goals, manufacturing SMEs have to consider potential constraints (i.e., hindering factors to adoption). Specifically, constraints can be related to technological, environmental, organizational, and actor readiness. The first two require responses from ImT providers, while the latter two require responses from the manufacturing SMEs themselves.

4.3.1 Technological readiness. The interviews revealed three constraints related to technological readiness. Hindering characteristics (i.e., complexity and compatibility) of ImT are often mentioned as a constraint for manufacturing SMEs when considering the adoption of ImT. Currently many solutions based on $\operatorname{ImT}$ require dedicated infrastructure and hardware, which hinders rapid installation and adoption as SMEs often cannot afford such investments. Many respondents would prefer solutions that run on existing devices (e.g., mobile phones), rather than requiring purchase of additional specialist equipment. In addition, VR solutions often require a connection to a computer and a physical power supply, which hampers their portability. Also, data security and privacy concerns can be a key constraint for manufacturing SMEs that are considering implementing ImT, as ImT solutions often contain sensitive data and content about the SME and its products. Concerns that the data transferred between the different parties may not be secure and the 
lack of SME-internal expertise to ensure data security itself, constrain adoption of ImT. In addition, when using ImT, the data is often provided to others in realtime, raising concerns about the continuous surveillance and monitoring of employees, whether intentional or not. Furthermore, the interviews indicate weak form factors, such as discomfort for employees wearing devices when using $\operatorname{ImT}$ (e.g., glasses or helmets), constrain ImT adoption: "At some point the health and safety unit asks whether employees can wear such heavy glasses for so long" [I14].

To address these constraints, the study indicates three responses that providers of ImT should take. Firstly, they should focus on the development of simple product features to reduce the complexity to adopters. Also, the implementation of ImT themselves by SMEs should be easy and compatible with existing hardware, for instance by leveraging devices such as tablets and smartphones. Secondly, providers of ImT should focus on GDPR compliance with respect to their solutions to proactively address data security concerns. Providers should also enhance form factors to improve convenience for, and thus acceptance by users of ImT. Providers of ImT should not only work on software improvements and new functionalities, but also on the wearability and comfort.

4.3.2 Environmental readiness. This research identified three constraints related to environmental readiness. Many manufacturing SMEs experienced significant infrastructure and bandwidth challenges when they attempt to use ImT. Specifically, unstable internet connection and insufficient bandwidth constrain manufacturing SMEs employees' use of ImT for remote collaboration or maintenance, as employees are not able to establish a stable connection with their international colleagues. Enterprises with limited financial resources, such as SMEs, are often using regular internet connections, as opposed to dedicated high speed connections with built in redundancies that larger enterprises have access to: "You need $5 G$ and the associated infrastructure. This is an important issue in general, and with VR and $A R$, the infrastructure must be available" [I03]. Moreover, legal and safety concerns are a key constraint that can hinder the adoption of ImT. Employee safety is a key concern for the manufacturing industry. In addition, technology unavailability is referenced as a constraint for the adoption of ImT, as most solutions manufacturing SMEs can afford are off the shelf and not necessarily tailored to their specific use cases.

To address these constraints, providers of ImT should develop data-light solutions that match the existing infrastructure of most manufacturing SMEs.
Some ImT solutions may include too many features for the adopting SMEs, that would favor data-light and thus, more reliable solutions, given their infrastructure situation. Moreover, ImT providers should enhance security controls and lobby governments to revise laws. Providers of ImT have to enhance the security provided to SMEs by improving solutions technologically and by urging governments to revise regulations that cover potential security issues of ImT. Governments also play a key role in providing the required internet and bandwidth for SMEs.

4.3.3 Organizational readiness. Regarding organizational readiness, the study reveals three major constraints. Many interview partners expressed concerns about the lack of required data and content that is needed to effectively establish ImT solutions. Specifically, the simulated environments often require the user to capture the content from the real world, generate the data, and map it into a 3D model. This is a constraint for manufacturing SMEs, which often have little programming and graphical experience, or cannot afford the acquisition of these skills on a contract basis. Strongly related to this is the financial constraint that also causes considerable tension within SMEs, as they do not have large budgets to spend on innovations. This often pushes SMEs towards solutions which are off the shelf and low-tech, but at a lower price point and ideally easier to implement and maintain. Moreover, lacking foresight and strategic planning hinders ImT implementation. Manufacturing SMEs that decide on investing in ImT are required to analyze the full spectrum of benefits that can be provided, even though it can take several years to see significant benefits from the implementation: "Many people in companies, especially the decision-makers, have NO idea about the technology, have never tried it themselves or have never come across VR or AR in their training" [I03].

The aforementioned data- and content-related constraints can be addressed by manufacturing SMEs developing a more conscientious approach to data, and also by building internal skills. Manufacturing SMEs are advised to invest in the collection, preparation and processing of machine data and related content to have sufficient data for virtualization and augmentation. Moreover, agile decision making was mentioned as a response by SMEs as it enables them to overcome organizational constraints and make use of ImT rapidly. The lack of corporate structures and processes in SMEs often creates an environment that is very receptive and appreciative of innovations once an investment decision is made. Moreover, SMEs are often characterized by a flexible work mindset and the ability to enhance and extend it to their 
manufacturing environment. As such, decisions can be made rapidly and tailored to the SME's individual circumstances, usually without time-intense communication cascades. Furthermore, SMEs should consider investments in ImT to address the trend in ImT that is gaining momentum in manufacturing. However, given their limited resources, the investment must be validated by a strong business case and provide a clear advantage over low-tech options such as video-conferencing tools.

4.3.4 Actor readiness. The study indicates two constraints in the context of actor readiness. Firstly, manufacturing SMEs may experience resistance to change by their employees when it comes to the implementation of new technologies in general and the use of ImT in particular. Employees may experience difficulties in using ImT in a way that complements existing processes, resulting in concerns that must be addressed: "You definitely need at least one or two who are $1000 \%$ convinced of it [...] and can then take over the lead and convey the enthusiasm to the other employees" [I08]. Secondly, a lack of relevant skills related to $\operatorname{Im} T$ is a constraint for the set up and uptake of affordances of the associated ImT. Employees of SMEs are often required to fulfil many roles, including business critical functions, and do not have the skills, nor the time to obtain the technical skills to identify, implement, and maintain ImT.

In order to address the constraints, manufacturing SMEs can consider the following three responses: Firstly, there should be a strong focus on merging new solutions with existing processes. Existing processes are already embedded and understood by the organization, and enhancing these processes is much more likely to be positively received by employees than a process redesign. Secondly, due to the often lack of ImT skills in manufacturing SMEs, they should consider outsourcing of solution development to external providers. There is already a strong reliance on providers to implement the ImT, usually as an off the shelf solution, rather than establishing in-house capabilities to develop and manage a customized solution internally. Thirdly, manufacturing SMEs can train and up-skill their employees in the use of ImT to increase their familiarity with these solutions. This requires not just traditional systems training, but also safety and risk assessment due to the system's ability to alter the employees' reality.

\section{Discussion}

ImT provide great opportunities for manufacturing SMEs, especially for remote collaboration and maintenance. To answer our research questions, this research examines the affordances provided by ImT to manufacturing SMEs, how the constraints of ImT may hinder their adoption, and how manufacturing SMEs and providers of ImT can respond to such constraints. The research questions are addressed by drawing on prior literature on the affordances of ImT, viewing this as an example of an activity-based approach which puts a strategic decision into practice. Thus, the research confirms the previously described affordances of ImT and further specifies them for SMEs in a manufacturing context, taking manufacturing SMEs' organizational and managerial characteristics into account. Additionally, the conceptualization of constraints is expanded to include them as inhibiting factors for technology adoption along the four dimensions of technological, environmental, organizational, and actor readiness. In addition, this research outlines the responses that can be taken by providers of ImT and manufacturing SMEs to address the respective constraints, taking advantage of the ImT's affordances.

\subsection{Research contributions}

The findings make two significant contributions to existing research on ImT's affordances and constraints, and provide insights into how SMEs strategize in the manufacturing industry: Firstly, they provide a more nuanced understanding of affordances and constraints that impact the adoption of ImT in manufacturing SMEs as a strategic response to environmental changes. Prior research has identified a variety of affordances of ImT which are applicable for different use cases and actors [2, 7]. However, different actors (e.g., enterprises of different sizes) experience different affordances and constraints with the same technology [22]. Moreover, the affordances of ImT have mostly been studied in isolation, largely omitting constraints that may occur at the same time and are known to hinder technology adoption [21, 22]. Therefore, by uncovering and contrasting the affordances and constraints of ImT for manufacturing SMEs, this research contributes to a better understanding of the driving and inhibiting factors for the adoption of ImT by manufacturing SMEs.

Secondly, this research extends SaP literature by investigating the adoption of ImT by SMEs as a strategic response to environmental changes. $\mathrm{SaP}$ is an approach that examines the link between strategy and the actions by individual actors $[17,23]$. This study extends the existing research by examining SMEs, which show a strong blend between strategy and practice, often making them indistinguishable due to SMEs' flat hierarchy, comparably lean organizational structure, and fast response to emerging trends and 
technologies $[10,13,14]$. This is also the case with the adoption of ImT which represents an institutional shift that SMEs need to respond to. Moreover, this research expands SaP's core focus on enterprise-internal actors to include the role of the providers of $\operatorname{ImT}$ and incorporate constraints which inhibit the realization of the affordances associated with the strategy (i.e., use of ImT) regarding providers, and the adopting SMEs. By examining strategizing in SMEs, including the consideration of their specific characteristics and their tight interrelation to their providers, this research provides a better understanding of the adoption of ImT as a strategic response, and contributes to an enhanced view of SaP in SMEs.

Moreover, the results are of considerable interest for practitioners (i.e., manufacturing SMEs' executives) who are responsible for strategizing and the adoption of ImT. While ImT are usually characterized by their functionalities and affordances, prior research indicates a technology's constraints have a significant influence on its adoption [21, 22]. Thus, manufacturing SMEs' executives should evaluate ImT not only in terms of the affordances (e.g., collaboration and communication enhancement), but also in terms of the constraints regarding environmental and technological readiness, as well as their SMEs' organizational and actor readiness. By doing so, manufacturing SMEs can identify the required responses to address the existing constraints and take advantage of the ImT's affordances.

Lastly, this study provides a practical set of affordances, constraints, and responses which are relevant for manufacturing SMEs to improve the organizational adoption of ImT. This research provides an overview of constraints and responses that can be considered by manufacturing SMEs to evaluate the implementation of ImT in their organization. Thus, SMEs can implement the responses suggested by their peers in this study if they experience any of the constraints identified in the study. Additionally, SMEs can discuss the reasons for the differences in their orientations and successes in overcoming constraints by reviewing the responses identified in this study.

\subsection{Limitations and future research}

Despite its contributions, this study is not without limitations. Firstly, the interview sample was limited in size and restricted to SMEs with German headquarters. Future research should investigate the affordances and constraints of ImT in manufacturing SMEs in other geographies. We also call for further research to validate the responses of SMEs and providers to identify additional constraints and provide more detailed guidance to address them. The results of this study could also be extended by a longitudinal study, analyzing the effect of responses on the ImT adoption after overcoming the constraints.

Furthermore, future research should investigate ImT adoption in industrial contexts that extend beyond manufacturing. Affordances in areas such as virtual training, learning, and collaboration are important for SMEs in all industries. Understanding the industryspecific constraints and identifying the corresponding responses could help SMEs in different industries to adopt ImT effectively.

\section{Conclusion}

Manufacturing SMEs can strongly benefit from affordances of ImT adoption, enhancing internal and external collaboration, reducing their travel efforts, and ultimately minimizing costs. However, adoption rates are still low, and prior research has focused on the investigation of affordances rather than on constraints and respective responses to the constraints. Building on technology affordances and constraints theory and $\mathrm{SaP}$, the research extends existing research on technology adoption and uncovers four areas of constraints that inhibit the adoption of $\operatorname{ImT}$ by manufacturing SMEs. Moreover, the study reveals the responses that can be taken by manufacturing SMEs and ImT providers to address these constraints and ultimately take advantage of the affordances of ImT.

\section{References}

[1] Milgram, P. and F. Kishino, "A Taxonomy of Mixed Reality Visual Displays". IEICE Transactions on Information Systems, 1994. E77-D(12): p. 1321-1329.

[2] Steffen, J., et al., "Framework of Affordances for Virtual Reality and Augmented Reality". Journal of Management Information Systems, 2019. 36(3): p. 683729.

[3] International Data Corporation. Worldwide Spending on $A R$ and VR Forecast to Deliver Strong Growth Through 2024. 2020 [cited 25.05.21]. Available from: www.idc.com/getdoc.jsp?containerId=prUS47012020.

[4] Winkler, N., et al. "Lose Yourself in VR: Exploring the Effects of Virtual Reality on Individuals' Immersion". in 53rd HISCSS. 2020. Hawaii.

[5] Elliott, A., B. Peiris, and C. Parnin, Virtual Reality in Software Engineering: Affordances, Applications, and Challenges, in 37th IEEE International Conference on Software Engineering. 2015, IEEE: Florence, Italy.

[6] Shin, D.-H., "The Role of Affordance in the Experience of Virtual Reality Learning". Telematics and Informatics, 2017. 34(8): p. 1826-1836.

[7] Kammler, F., et al. "How Do We Support Technical Tasks in the Age of Augmented Reality? Some Evidence from Prototyping in Mechanical Engineering". in 40th ICIS Proceedings. 2019. Munich, Germany. 
[8] Steffen, J., et al. "The Missing Framework for Virtually Assisted Activities". in 38th ICIS. 2017. Seoul.

[9] Berg, L.P. and J.M. Vance, "Industry Use of Virtual Reality in Product Design and Manufacturing: A Survey". Virtual Reality, 2017. 21(1): p. 1-17.

[10] Kraus, S., et al., "The Economics of COVID-19: Initial Empirical Evidence on How Family Firms in Five European Countries Cope With the Corona Crisis". International Journal of Entrepreneurial Behavior \& Research, 2020. 26(5): p. 1067-1092.

[11] European Commission, Annual Report on European SMEs 2018/2019. 2019, Luxembourg: Publications Office of the European Union.

[12] IfM. Family Business Defintion of the IfM Bonn. 2021 [cited 27.05.21]. Available from: www.ifm-bonn.org/en/ definitions/family-business-definition-of-the-ifm-bonn.

[13] Xi, J., et al., "Mapping the Field of Family Business Research: Past Trends and Future Directions". International Entrepreneurship and Management Journal, 2015. 11(1): p. 113-132.

[14] Heidt, M., J. Gerlach, and P. Buxmann, “Investigating the Security Divide between SME and Large Companies: How SME Characteristics Influence Organizational IT Security Investments". Information Systems Frontiers, 2019. 21(6): p. 1285-1305.

[15] Chan, Y.E., R. Krishnamurthy, and C. Desjardins, "Technology-Driven Innovation in Small Firms". MIS Quarterly Executive, 2020. 19(1): p. 39-55.

[16] Eckhardt, A., et al., "Three Stages to a Virtual Workforce". MIS Quarterly Executive, 2018. 18(1): p. 19-35.

[17] Jarzabkowski, P., J. Balogun, and D. Seidl, "Strategizing: The Challenges of a Practice Perspective". Human Relations, 2007. 60(1): p. 5-27.

[18] Peppard, J., R.D. Galliers, and A. Thorogood, "Information Systems Strategy as Practice: Micro Strategy and Strategizing for IS". Journal of Strategic Information Systems, 2014. 23(1): p. 1-10.

[19] Eden, R., et al., "Digital Transformation Requires Workforce Transformation”. MIS Quarterly Executive, 2018. 18(1): p. 1-17.

[20] Chan, Y.E., R. Krishnamurthy, and C. Desjardins, "Technology-Driven Innovation in Small Firms". MISQ Executive, 2020. 19(1): p. 39-55.

[21] Volkoff, O. and D.M. Strong, "Critical Realism and Affordances: Theorizing IT-Associated Organizational Change”. MIS Quarterly, 2013. 37(3): p. 819-834.

[22] Leonardi, P.M., "When Flexible Routines Meet Flexible Technologies: Affordance, Constraint, and the Imbrication of Human and Material Agencies". MIS Quarterly, 2011.35(1): p. 147-167.

[23] Jarzabkowski, P. and A.P. Spee, "Strategy-as-Practice: A Rreview and Future Directions for the Field". International Journal of Management Reviews, 2009. 11(1): p. 69-95.

[24] Sutherland, I. "A Head-Mounted Three Dimensional Display". in Proceedings of the 1968 Fall Joint Computer Conference AFIPS. 1968. San Francisco, California.

[25] PwC. For Us Manufacturing, Virtual Reality is for Real. 2016 [cited 03.06.21]. Available from: www.pwc.com/ us/en/industrial-

products/publications/assets/augmented-virtual-realitynext-manufacturing-pwc.pdf.

[26] Brooks, F.P., "What's Real About Virtual Reality?" IEEE Computer Graphics and Applic., 1999. 19(6): p. 16-27.

[27] Gibson, J.J., A Theory of Affordances, in Perceiving, Acting and Knowing: Toward an Ecological Psychology, R. Shaw and J. Bransford, Editors. 1977, Erlbaum Associates: Hillsdale, NJ. p. 67-82.

[28] Majchrzak, A. and M.L. Markus, Technology Affordances and Constraints Theory of MIS, in Encyclopedia of Management Theory, E.H. Kessler, Editor. 2013, SAGE Publications: Thousand Oaks. p. 832-836.

[29] Li, X., A. Rai, and G. Krishnan, "Designing CostEffective Telemedicine Camps for Underprivileged Individuals in Less Developed Countries: A Decomposed Affordance-Effectivity Framework". Journal of the Association for IS, 2020. 21(5): p. 12791312.

[30] Markus, M.L. and M. Silver, "A Foundation for the Study of IT Effects: A New Look at DeSanctis and Poole's Concepts of Structural Features and Spirit". Journal of the Association for IS, 2008. 9(10): p. 609632.

[31] Yin, R.K., Case Study Research: Design and Methods. 2nd ed. 1994, Los Angeles: SAGE Publications.

[32] Sarker, S., et al., "Learning from First-Generation Qualitative Approaches in the IS Discipline: An Evolutionary View and Some Implications for Authors and Evaluators (PART 1/2)". Journal of the Association for IS, 2018. 19(8): p. 752-774.

[33] Huber, G.P. and D.J. Power, "Retrospective Reports of Strategic-Level Managers: Guidelines for Increasing Their Accuracy". Strategic Management Journal, 1985. 6(2): p. 171-180.

[34] Eisenhardt, K. and M. Graebner, "Theory Building From Cases: Opportunities And Challenges". Academy of Management Journal, 2007. 50(1): p. 25-32.

[35] Etikan, I., S.A. Musa, and R.S. Alkassim, "Comparison of Convenience Sampling and Purposive Sampling". American Journal of Theoretical and Applied Statistics, 2016. 5(1): p. 1-4.

[36] Myers, M. and M. Newman, "The Qualitative Interview in IS Research: Examining the Craft". Information and Organization, 2007. 17(1): p. 2-26.

[37] Neergaard, H. and J.P. Ulhøi, Handbook of Qualitative Research Methods in Entrepreneurship. 2007, Cheltenham; Northampton: Edward Elgar Publishing.

[38] Miles, M.B. and A.M. Huberman, Qualitative Data Analysis: An Expanded Sourcebook. 2nd ed. 1994, Thousand Oaks; London; NewDelhi: SAGE Publications.

[39] Saldana, J., The Coding Manual for Qualitative Researchers. 2nd ed. 2013, Los Angeles, London, New Delhi, Singapore, Washington DC: SAGE Publications.

[40] Boddy, C.R., "Sample Size for Qualitative Research". Qualitative Market Research: An International Journal, 2016. 19(4): p. 426-432. 